\title{
APPLICATION OF NON-LINEAR ANALYSIS TECHNIQUES TO SIGMA-DELTA MODULATORS
}

\author{
Ronan Farrell and Orla Feely
}

\begin{abstract}
Introduction
Sigma-delta modulators are based upon the idea of using feedback to improve the effective resolution of a coarse quantiser, commonly a 1-bit quantiser [1]. The modulator is a discrete-time system and thus suited to VLSI implementation. The most basic sigma-delta modulator is the first order, or single-loop, modulator. This modulator consists of a single feedback loop from the quantiser to the input of a discrete-time integrator. It can be shown that the effect of the integrator and the feedback loop is to null the quantisation noise at zero frequency, and so for low-frequency inputs it is possible to obtain a high signal-to-noise ratio at the output. This noise-shaping function is one of the major advantages of sigma-delta modulation. It is possible to enhance this noiseshaping by increasing the number of integrators and adding additional feedback loops from the quantiser to the inputs of these new integrators. In this manner it is possible to obtain very good noise performance at low frequencies.
\end{abstract}

Sigma-delta modulators can be categorised by the number of integrators in the system and by the number of feedback loops from the quantiser. A sigma-delta modulator with only one feedback loop to the input of the transfer function is commonly called a single-loop modulator (Figure 1). Another more recent form of classification is based on the filtering function of the modulator. It is possible to design the transfer function such that there is a null in the noise power at a frequency other than zero, obtaining faithful conversion of high-frequency narrowband signals. These modulators are called band-pass modulators [2] and correspondingly the traditional form is called low-pass sigma-delta modulation (Figure 2).

Due to the noise-shaping ability of sigma-delta modulators and the linearity of the output with respect to the input, they have become popular as the modulation scheme for oversampled analogto-digital and digital-to-analog converters [1]. A sigma-delta modulator requires only simple analog components that are insensitive to circuit imperfections. Complex digital circuitry is then used to produce a high precision digital representation from this low-noise coarse quantisation.

In the past sigma-delta modulators have been analysed using linear analysis techniques [3]. In these approaches the quantiser is replaced by an additive white noise source and then standard linear systems analysis is applied. This approach can provide good estimates of noise performance but it is unable to explain much of the behaviour of sigma-delta modulators, especially such phenomena as instability, integrator spans, quantiser switching frequency, idle tones, strong limit cycle behaviour and chaos, which are inherently non-linear. To examine these problems it will be necessary to utilise some of the many non-linear analysis techniques now available. A full rigorous non-linear analysis of these systems using any one technique would be very difficult, if not impossible. An alternative is to identify specific problems, such as instability, and to apply the most suitable non-linear analysis technique to the problem. Three main approaches have been applied to the non-linear analysis of sigma-delta modulators: spectral analysis (noise and signal performances), geometric analysis (stability analysis and integrator spans) and non-linear dynamics (limit cycle behaviour).

Ronan Farrell and Orla Feely are with the Electronic and Electrical Engineering Department of University College Dublin, Belfield, Ireland. Emails: Ronan.Farrell@ucd.ie and Orla.Feely@ucd.ie 


\section{Spectral Analysis}

This form of analysis was pioneered by Gray [4, 5] and was extended by He, Kuhlmann and Buzo [6]. In [4] Gray considered the first order low-pass modulator, which is defined by the single difference equation

$$
U_{n+1}=U_{n}+x_{n}-\operatorname{sgn}\left(U_{n}\right)
$$

where $U$ is the state variable and $x_{n}$ is the input. Gray proved the equivalence between the first order modulator with constant input and the circle map. The circle map is a well known function in non-linear dynamics and ergodic theory. Using this equivalence it was possible to explain some of the basic behaviour of the system, for example, the fact that rational inputs lead to a limit cycle. Gray, through the solution of a system of non-linear equations, was also able to determine the true quantisation noise behaviour and then proceeded to develop the overall noise spectrum. It was shown that the assumption of white quantisation noise was incorrect, as there was a strong correlation between the input and the output bit pattern. This work was then extended by Gray and He et al to cover double-loop systems with both constant and sinusoidal inputs.

The extension of this work to systems other than the first order single-loop modulator required the assumption that the quantiser never overloads. This is only applicable to systems with multi-bit quantisers, as these may be designed not to overload. Another drawback to this approach is the difficulty in extending the analysis to systems with more than two loops. This approach is limited to noise spectra but to date it is the best approach available for this analysis, other than linearising the system. One important result of this analysis was the identification of the optimal FIR filters for the output of a sigma-delta modulator, and that the commonly used sinc filters are close to optimal.

\section{Geometric Analysis}

In this approach the trajectories of the integrator outputs are analysed. This approach is primarily concerned with stability and identifying the integrator spans of second order systems $[7,8]$ and some third order systems [9]. This approach depends on identifying regions in state space (where the state variables are the integrator outputs) where the mapping function of the modulator is affine. Within these regions the trajectories of the outputs of the integrators lie on parabolae (Figure 3). The difficulty lies in identifying the behaviour of the trajectories upon crossing between the piecewise-linear regions. The approach promoted independently by Hein and Zakhor [7] and Wang [8] concentrated on the second order low-pass system and considered the trajectories as continuous curves. They showed that for all inputs of constant value in the range $(-1,1)$ there was some maximum size of parabola beyond which the trajectories collapsed down to be within this maximum parabola. In this manner they proved that the second order modulator was stable for inputs within the range $(-1,1)$ and they were also able to develop strong bounds for the maximum value that the integrator outputs may reach (Figure 4).

Pinault and Lopresti extended this approach to include the class of inputs consisting of a constant input of magnitude less than 1 and of an arbitrary sum of finite amplitude sinusoids [10]. This was the first time it was shown that the second order modulator was stable for sinusoidal inputs. The bounds produced are interesting but not very useful as they tend to infinity as frequency tends to zero.

A more recent approach by Farrell and Feely [11] considers the trajectories as discrete points along a parabolic curve. This complicates the analysis of the behaviour of the trajectories in the transition region between the half-planes. It is possible to remove the time component from the entry positions of the trajectories in these transition regions. Using this technique all possible parabolae can be identified in the new half-plane. If this approach is used for both half-planes, an iterative approach can be developed to identify the maximum size of the parabolae. This is similar in principle to that of Hein, Zakhor and Wang, but as it acknowledges the discrete nature of the 
trajectories much tighter bounds can be achieved (Figure 4). One of the results of this more detailed analysis is that the range of all possible values for the integrator outputs can be determined (Figure 5) and the existence and stability of limit cycle behaviour can be defined [11]. This approach has been extended to include the case of chaotic sigma-delta modulators. Chaotic modulators may be obtained by placing one of the poles of the integrators of the second order modulator outside the unit circle. This has been suggested as a means of reducing the problem of idle tones, but has a penalty in dynamic range of input and stability. Current work on this approach has shown that it can be extended to cover the full range of time-varying inputs including sinusoidal and square-wave inputs. Second order modulators may go unstable under certain timevarying inputs. Linear analysis can not determine what types of inputs will result in instability. A future avenue for development would be to use the approach of Farrell and Feely to determine the conditions under which the second order low-pass sigma-delta modulator may become unstable.

\section{Non-Linear Dynamics}

The principles of non-linear dynamics were first applied to sigma-delta modulators by Feely and Chua [12]. The strength of non-linear dynamics is that it provid: insightful information into the behaviour of non-linear systems and thus can give useful inform. on about limit cycle effects and idle tones. Gray had shown that limit cycles arise from rational inputs. Feely and Chua, using symbolic analysis, proved that as the integrators become less perfect, the value of inputs close to the simplest rationals, for example $0,0.5,0.25$, fall into the limit cycle behaviour associated with those rationals, leading to a reduction in accuracy. This effect is best shown in a plot of input against output which results in the well-known "devil's staircase" (Figure 6).

Feely [13] first analysed the band-pass sigma-delta modulator with the state equation

$$
U_{n}=2 U_{n-1} \cos \theta-U_{n-2}+2 \cos \theta\left(x_{n-1}-\operatorname{sgn}\left(U_{n-1}\right)\right)-\left(x_{n-2}-\operatorname{sgn}\left(U_{n-2}\right)\right)
$$

Feely identified the fixed and low order period points of this map. It was then possible to show that if a trajectory started close to these fixed points the trajectories would move elliptically around these points. Outside these regions, the behaviour of the trajectories becomes fractal in nature. An example is shown in Figure 7 where $\cos \theta=-0.158532$ and with zero input. This work was then extended to consider sinusoidal inputs [14]. The importance of these results is that a single ellipse or a finite number of ellipses is equivalent to the limit cycle behaviour in the low-pass modulators. If the systems is capable of locking into an ellipse or a finite number of ellipses, then a loss of accuracy may result. This potential loss of accuracy can be avoided only by identifying the conditions under which such behaviour can arise. This would not be possible using any linear technique.

\section{Conclusion}

The behaviour of even the simplest sigma-delta modulator is complex and difficult to analyse. Non-linear analysis techniques can provide an understanding of some of the unexpected behaviour of these apparently simple looking systems. No one approach will yield all the required results so it is important to have an understanding of which approach is useful for the specific problem under investigation. The various approaches discussed in this paper are all useful in their own way. They can be extended to some degree to consider other tasks but primarily spectral analysis is suitable for noise performance and spectra, geometric analysis for bounds and stability and non-linear dynamics for limit cycle behaviour and other behavioural effects.

This paper has concentrated on the sigma-delta modulator but the discussion can be generalised to include other systems. The sigma-delta modulator shares many features with other discrete-time processes in digital signal processing, for example digital filters [15], and digital phase lock loops [16]. In recent years there has been significant growth in the application of non-linear analysis to many different systems. Many new techniques have being developed, enabling a deeper understanding of these complex systems to be obtained. 


\section{References}

1. Candy, J C and Temes G: "Oversampling Delta-Sigma Data Converters", IEEE Press, NY, 1991.

2. Schreier, $R$ and Snelgrove, $M$ : "Bandpass sigma delta modulation", Electronic Letters, Volume 25, pp. 1560-1561, 1989.

3. Ardalan, S and Paulos, J: "An analysis of Nonlinear behaviour in delta-sigma Modulators", IEEE Trans. Circuits and Systems, VOL. 34, pp. 593-603, 1987.

4. Gray R M: "Oversampled Sigma-Delta Modulation", IEEE Trans. Commun., VOL. COM-35, pp. 481-489, 1987.

5. Gray, R M: "Quantization Noise Spectra", IEEE Transactions on Information Theory. VOL. 36, pp. 1220-1244, 1990.

6. He, N, Kuhlmann, F and Buzo, A: "Double-Loop Sigma-Delta Modulation with dc Inputs", IEEE Trans. Commun., VOL. COM-38, pp. 487-495, 1990.

7. Hein, S, and Zakhor, A: "On the stability of sigma delta modulators," IEEE Trans. Signal Processing, Vol. 41, pp. 2322-2348, 1993.

8. Wang, H: "A geometric view of sigma delta modulations," IEEE Trans. CAS II - Analog and Digital Processing, Vol. 39, pp. 402-405, 1992.

9. Wang, H: "A study of sigma delta modulations as dynamical systems," PhD Thesis, Colombia University, New York, 1993.

10. Pinault, S and Lopresti, P: "On the behaviour of the double-loop sigma delta modulator," IEEE Trans. CAS II - Analog and Digital Processing, Vol. 40, pp. 467-479, 1993.

11. Farrell, R and Feely, O: "Bounding the Integrator Outputs of Second Order Sigma Delta Modulators" to be published IEEE Trans. CAS II - Analog and Digital Signal Processing.

12. Feely $O$ and Chua L O: "The effect of integrator leak in $\Sigma \triangle$ Modulation", IEEE Trans. Circuits and Systems, VOL. 38, pp. 1293-1305, 1991

13. Feely, O: "Nonlinear dynamics and chaos in sigma-delta modulation", Proceedings 3 rd International Workshop on Nonlinear Dynamics in Electronic Systems (NDES), 1995

14. Fitzgerald, D and Feely, O: "Bandpass sigma-delta modulation - an analysis from the perspective of non-linear dynamics", IEEE International Symposium on Circuits and Systems, VOL. III, pp. 146-149, 1996.

15. Chua, L O and Lin, T: "Chaos in digital filters", IEEE Trans. Circuits and Systems, VOL. 35, pp. $648-658,1988$

16. Rogers, A Naughton, D and Feely, O: "Nonlinear Analysis of a digital phase-locked-loops", European Conference on Circuit Theory and Design, pp. 121-126, 1997.

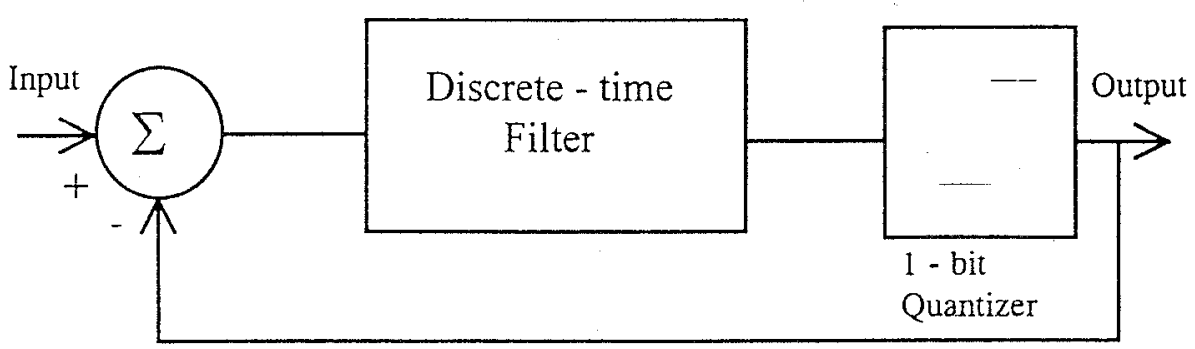

Figure 1: Single-loop sigma-delta modulator with 1-bit quantiser. 

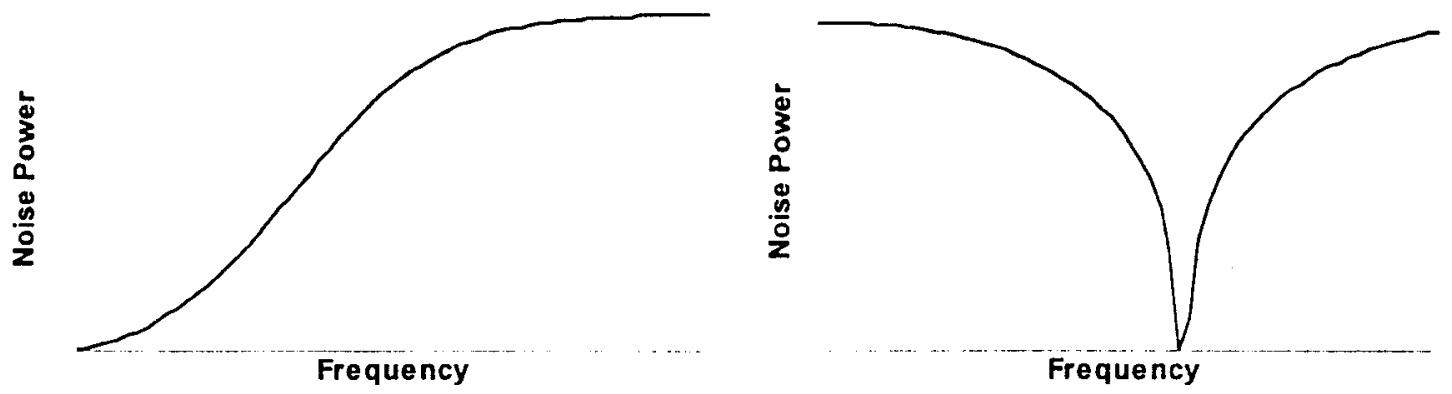

Figure 2: The noise performance of low-pass and band-pass modulators respectively.

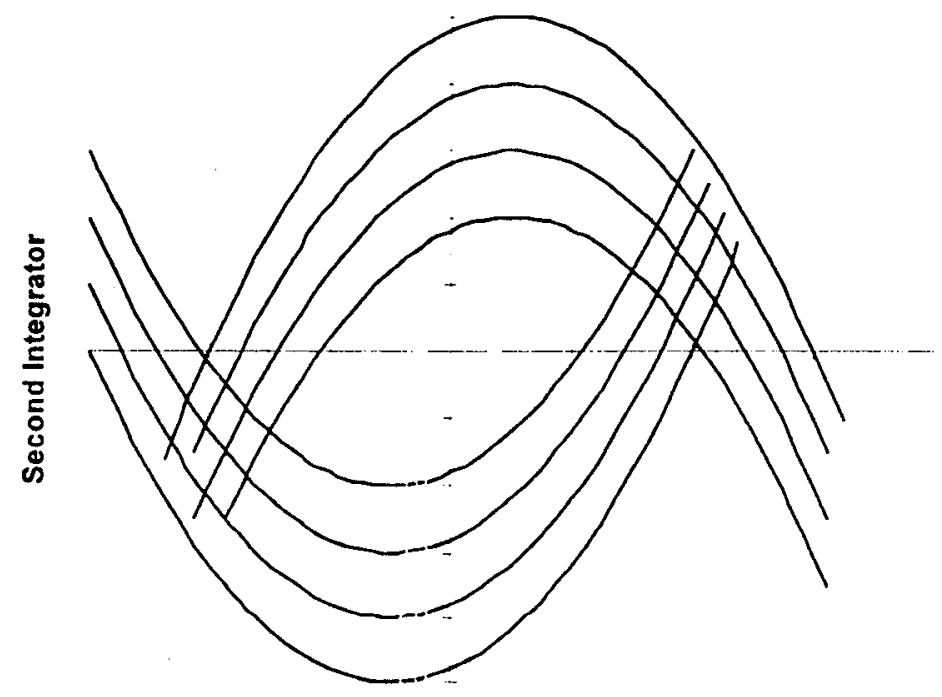

First Integrator

Figure 3: The parabolic trajectories of the outputs of the integrators and their transition regions

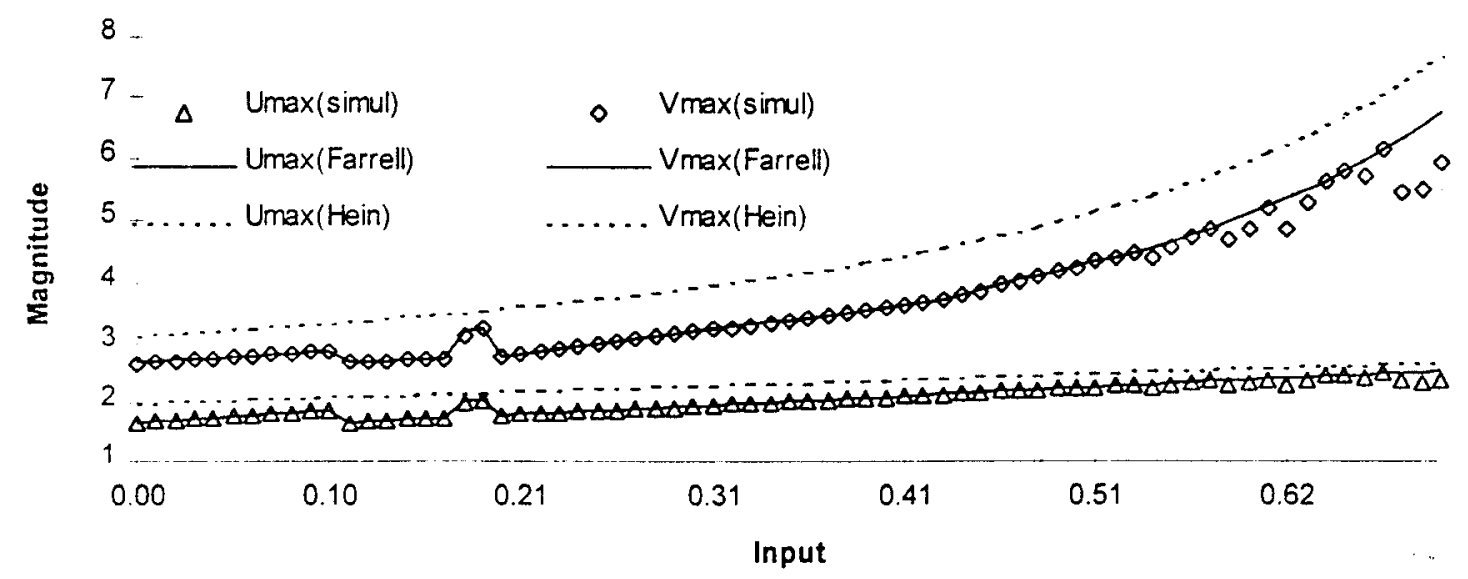

Figure 4: A comparison of the bounds obtained by Hein and Zakhor [7], by Farrell and Feely [8] and from the maxima obtained from simulation. 


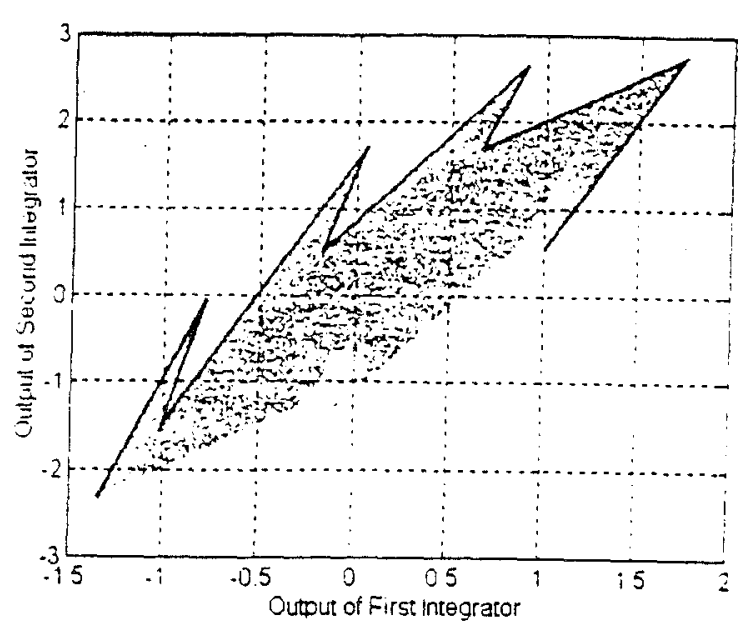

Figure 5: A plot of the values that the integrator outputs may take and the boundaries on these values that can be determined using the approach of [11]

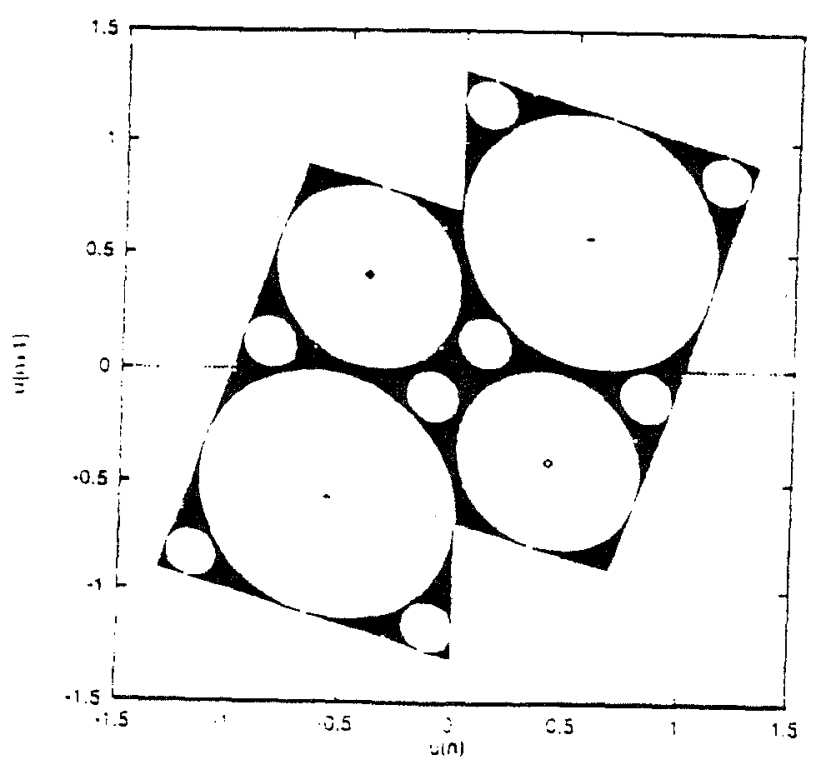

Figure 7a: A plot of 100.000 points of the traiectory of equation (2) with tixed points $1-1$ and period 2 points $(0)$ included.

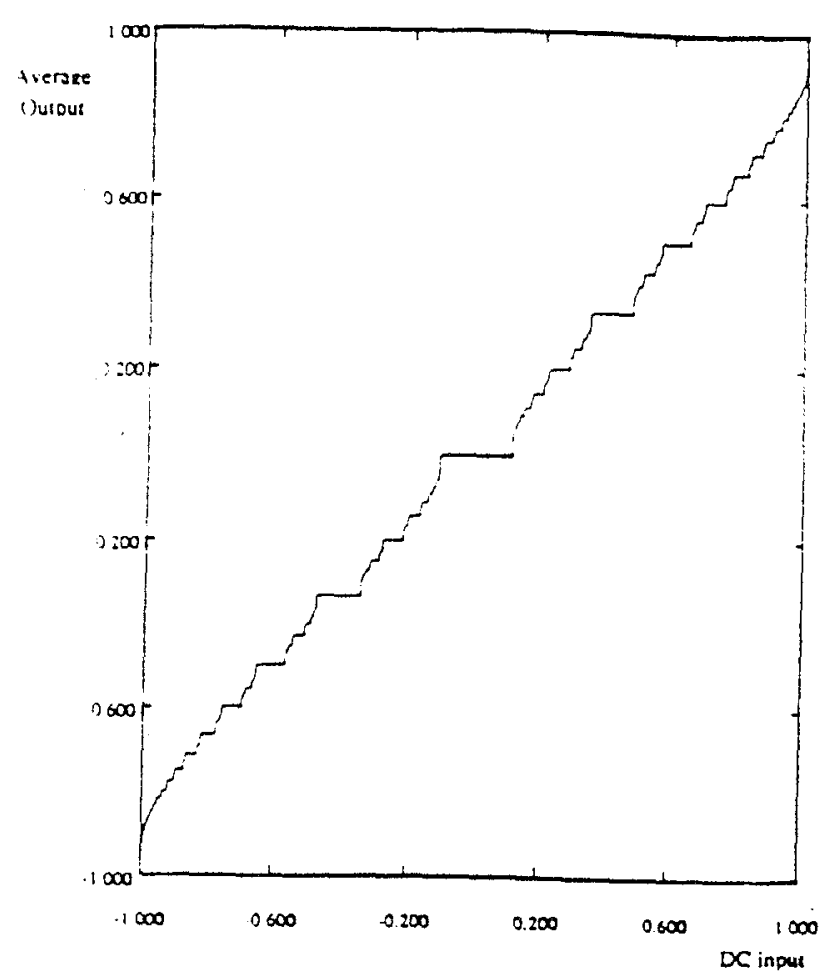

Figure 6: A graph of the average output versus input for a leaky first order $\Sigma \Delta$ modulator

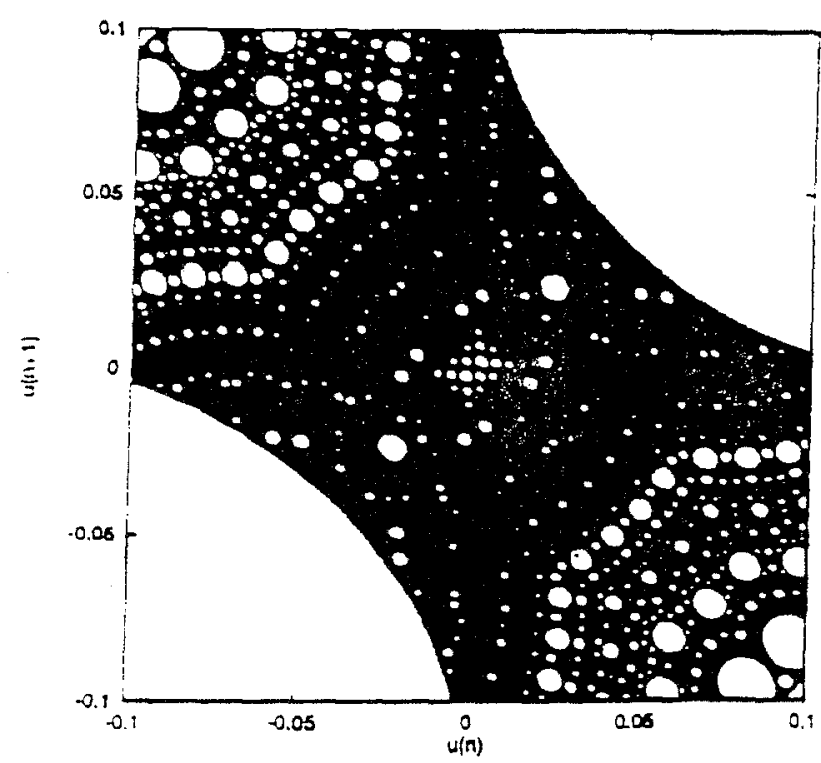

Figure 7b: Magnification of the centre section of Figure $7 \mathrm{a}$. 